\title{
FLEXIBLE FRAMEWORK FOR MODELING WATER CONVEYANCE NETWORKS
}

\author{
João Miguel Lemos Chasqueira Nabais \\ IDMEC, Department of Systems and Informatics, Escola Superior de Tecnologia de Setúbal \\ Campus do IPS, Estefanilha 2910-761 Setúbal, Portugal \\ joao.nabais@estsetubal.ips.pt \\ José Duarte \\ Departamento de Informática, Universidade de Évora, Rua Romão Ramalho 59, 7000-671 Évora, Portugal \\ m5642@alunos.uevora.pt \\ Miguel Ayala Botto \\ IDMEC, Instituto Superior Técnico, Technical University of Lisbon, Av. Rovisco Pais, 1049-001 Lisboa, Portugal \\ ayalabotto@ist.utl.pt \\ Manuel Rijo \\ NuHCC - Hydraulics and Canal Control Center, Universidade de Évora \\ Pólo da Mitra Apartado 94, 7002-554 Évora, Portugal \\ rijo@uevora.pt
}

\begin{abstract}
Keywords: Water Delivery Canals, Modeling, Network Transportation Systems, Leak Detection, Fault Tolerant Control.
Abstract: $\quad$ A flexible framework for modeling different water conveyance networks is presented. The network is modeled using a linear canal pool model based on the Saint-Venant equations to describe transportation phenomenon occurring in open channels. This model is used as a link to connect different nodes defined by gates or reservoirs. The linear pool model has interesting features namely the pool axis monitoring, the inflow along the pool axis and the ability to consider different boundary conditions. Based on these characteristics canal pool observers for leak detection and localization can be developed. It is shown that based on a finite difference scheme a good performance is obtained for low space resolution. The modeling framework is validated with experimental data from a real canal property of the Évora University. This is a challenging configuration due to its strong canal pool coupling.
\end{abstract}

\section{INTRODUCTION}

Water is vital for mankind way of life. It is used for multiple purposes, essential for agricultural and industry, domestic use and even for recreation. Unfortunately, water is becoming a rare resource so contributions to increase water use efficiency are welcome.

As the water source is not always close to the end users there exists the need to create efficient system, or network, to execute the water conveyance. The water transportation problem is not exclusively dedicated to delivery water to users. Water has also to be transported to safety locations rendering the management of water systems a complex task. Complex water transportation systems span from small-scale to large distributed systems, as is the case of large rivers that often crosses different countries. Water trans- portation systems may be divided into the following categories (Negenborn et al., 2009),

Irrigations Canals: are responsible for transporting water often from a long distance source, to the users. The objective is to deliver the specified amount of water that is normally accomplished by controlling the water depth at the extraction localization;

Sewers Networks: these systems are responsible to transport the waste water (from houses or due to rain) to treatment plants. The objective is to avoid water contamination and also execute flood control;

Large Multi-purpose Reservoirs: the course of natural rivers are controlled by large dams in order to create a large water storage capacity that can be used for different objectives as power 
production, irrigation and flood control.

Open-channels models are mainly divided into physical principle models and data driven models (Zhuan and Xia, 2007). Physical principle models (Litrico and Fromion, 2009) are based on the process knowledge. In particular, for canal systems they are based on the Saint-Venant equations and on the geometrical and hydraulic system description. Naturally, the model performance is dependent on the system parameters accuracy, for high uncertainty parameter the performance decreases. They are also useful as they can give some physical insight in the control engineering design phase. Data driven models are based on identification tools leading to grey or black box models (Weyer, 2001). These methods require the physical existence of the canal but can produce a model with a high level of accuracy.

In this paper, a flexible framework for modeling water transportation networks is presented. The canal pool dynamics is the most relevant component as it is responsible for the transportation phenomena; in particular it is important to have a model capable of capturing the backwater, or by other words, the water profile along the pool axis, the wave translation and attenuation as well as the flow acceleration. Special features made available in this framework are due to the pool model (Nabais and Botto, 2011). In particular, with this canal pool model it is possible to,

- monitor the pool axis in water depth and discharge as the white box state space vector is composed by this information. In the presence of few sensors, the model can be used as an observer to predict the water depth along the pool axis and verify for example the danger of occurring overtopping. Using this ability the purposed model can also be used for the development of pool observers;

- execute outflows or inflows along the pool axis. With this feature it will be possible to proceed with the leak identification and localization on irrigation networks while for drainage systems it will be possible to account for additional water inflows in the case of torrential rains, for instance;

- choose the boundary condition as discharge imposed by an hydraulic structure, or water depth imposed by a reservoir, and model multipurpose reservoir systems using the pool model to connect different reservoirs;

- as the linear pool model is given as a state space representation, the dynamics are solved through matrices multiplications, with a low computational cost. This is of capital importance for large scale systems as computation effort may impose a limit to the largest tractable system dimension;
- extracting linear models for the local dynamics pool plus gate is straightforward, the boundary condition is replaced by the hydraulic linearized equation.

The paper has the following structure. Section 2 presents the experimental water delivery canal hold by the NuHCC - Hydraulics and Canal Control Center from the Évora University in Portugal. The water transportation system typical components - canal pools, discharge and water depth control structures and storage elements - are presented in section 3 . The canal pool dynamics is solved by linearization ans discretization of the Saint-Venant equations, the reservoirs are modeled as an integrator element and the gates are described by static relations. In section 4 a brief description of the developed MatLab-Simulink Toolbox-Library is given. In section 5 the purposed modeling framework is validated for the experimental canal. Here it is shown the reliability, accuracy and flexibility of the proposed hydraulic model for a wide range of inputs. Finally, in section 6 some conclusions are drawn.

\section{EXPERIMENTAL CANAL}

The experimental automatic canal, property of NuHCC is located in Mitra near Évora in Portugal. The canal is built with trapezoidal section (with $0.15 \mathrm{~m}$ bottom width and $1: 0.15$ side slope), a maximum height of $0.9 \mathrm{~m}, 145 \mathrm{~m}$ length and an average longitudinal bottom slope about 0.0015 . The canal works in closed loop to avoid water spillage, and the return flow to the reservoir is secured by a second canal (a traditional local upstream controlled canal Figure1). The water is pumped from the lower reservoir to the higher reservoir by two pumps. The canal inflow is controlled by an electrical MONOVAR valve located downstream the higher reservoir. The facility was designed for a maximum discharge of $0.090 \mathrm{~m}^{3} / \mathrm{s}$.

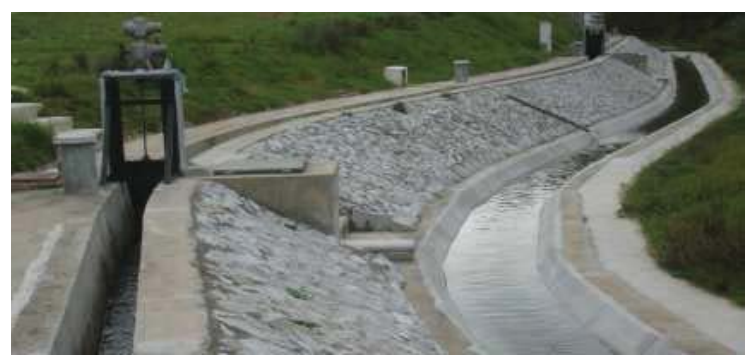

Figure 1: NuHCC canal property of Évora University.

In its basic configuration, the automatic canal is divided into four pools by three undershot gates and 
an overshot gate (vertical), this one located at the downstream canal section. Upstream each gate there exist an offtake, equipped with flow meter and an electrical butterfly, to allow water user extraction, and discharges into the return traditional canal. Float and counter-weight level sensors are distributed along the canal axis, three in each pool, allowing for water depth canal monitoring.

The facility has a 6 PLC network: five local, assigned to each gate and reservoirs, connected by a MODBUS network to the central master PLC. The master PLC communicates to the SCADA computer by a serial port RS232 interface. Recently, a SCADA-Controller Interface application has been developed (Duarte et al., 2011) allowing interaction with the facility through different environments as MatLab, C/C++ and GNU Prolog.

\section{WATER TRANSPORTATION NETWORKS}

Water conveyance networks are complex systems usually space distributed with a large dimension. Like other network systems they are composed by links and nodes. The link between nodes is accomplish by the water transportation element - the pool. The nodes establish the separation of different links and are represented by reservoir, gates or a combination of both.

\subsection{Pool Dynamics}

The flow dynamics in open channels is well described by the Saint-Venant equations (Akan, 2006), nonlinear partial differential equations of hyperbolic type capable of describing the transport phenomenon,

$$
\begin{aligned}
& \frac{\partial Q(x, t)}{\partial x}+T(x, t) \frac{\partial Y(x, t)}{\partial t}=0 \\
& \frac{\partial Q(x, t)}{\partial t}+\frac{\partial}{\partial x}\left(\frac{Q^{2}(x, t)}{A(x, t)}\right)+\ldots \\
& \ldots+g \cdot A(x, t) \cdot\left(S_{f}(x, t)-S_{0}(x)\right)=0
\end{aligned}
$$

where, $A(x, t)$ is the wetted cross section, $Q(x, t)$ is the water discharge, $Y(x, t)$ is the water depth, $T(x, t)$ is the wetted cross section top width, $S_{f}(x, t)$ is the friction slope, $S_{0}(x)$ is the bed slope, $x$ and $t$ are the independent variables. One approach to this complex problem is to linearize (1) (2) around a nonuniform steady configuration defined by $\left(Q_{0}, Y(L, 0)\right)$. To help future analysis is useful to consider the area deviation as $a(x, t)=T_{0}(x) y(x, t)$ and the state vector $\chi(x, t)=\left[\begin{array}{ll}q(x, t) & a(x, t)\end{array}\right]^{T}$. The linearized SaintVenant equations can be expressed in state space form as follows,

$$
\hat{A} \frac{\partial}{\partial t} \chi(x, t)+\hat{B}(x) \frac{\partial}{\partial x} \chi(x, t)+\hat{C}(x) \chi(x, t)=0
$$

where matrices $\hat{A}, \hat{B}(x)$ and $\hat{C}(x)$ are defined in (Litrico and Fromion, 2009). Numerical methods are known to introduce nonphysical behavior that is similar to the process physics and is not clear how to eliminate it (Szymkiewicz, 2010). The Preissmman scheme is the numerical method used to discretize the linearized Saint-Venant equations. The parameters $\phi$ and $\theta$ are weighting parameters for space and time respectively and vary between 0 and 1 . The index $i$ stands for section while index $k$ stands for time iteration.

The flow dynamics between two adjacent sections is described by (Nabais and Botto, 2011),

$$
\bar{A} x(k+1)+\bar{B} x(k)=\bar{B}_{w} w(k+1, k)
$$

where $x(k)=\left[\begin{array}{llll}q_{i}^{k} & a_{i}^{k} & q_{i+1}^{k} & a_{i+1}^{k}\end{array}\right]^{T}$ is the section $i$ state space vector and $w(k+1, k)=$ $\left[\begin{array}{ll}q_{o f f}^{k} & q_{o f f}^{k+1}\end{array}\right]^{T}$ represents the discharge perturbation between sections $i$ and $i+1$ where $q_{o f f}$ means the lateral outflow. The pool flow dynamic model is obtained interconnecting $N$ section models (4) leading to $2 N$ equations. The state space vector,

$$
\begin{array}{r}
\mathbf{X}(k)=\left[\begin{array}{lllll}
q_{1}(k) & a_{1}(k) & q_{2}(k) & a_{2}(k) & \ldots \\
\ldots & q_{n}(k) & a_{n}(k) & q_{n+1}(k) & a_{n+1}(k)
\end{array}\right]
\end{array}
$$

has dimension $2(N+1)$. To complete the model it is necessary to add boundary conditions. As the flow is considered subcritical one boundary condition for each end is introduced.

\subsubsection{Boundary Equations}

In the case of water discharge, when the pool is connected to an hydraulic structure as a gate or a pump, the boundary condition can be written as $u=q^{k+1}$ which means the model command signal is the next discharge value. In state space this is equivalent to,

$$
\left[\begin{array}{ll}
1 & 0
\end{array}\right]\left[\begin{array}{c}
q_{i}^{k+1} \\
a_{i}^{k+1}
\end{array}\right]+\left[\begin{array}{ll}
0 & 0
\end{array}\right]\left[\begin{array}{c}
q_{i}^{k} \\
a_{i}^{k}
\end{array}\right]=u
$$

A similar approach is done for the water depth boundary condition, when the pool is connected to an hydraulic structure or reservoir, written as $u=y^{k+1}$ so the model command signal is the next water depth value. In state space form this is equivalent to,

$$
\left[\begin{array}{ll}
0 & 1
\end{array}\right]\left[\begin{array}{l}
q_{i}^{k+1} \\
a_{i}^{k+1}
\end{array}\right]+\left[\begin{array}{ll}
0 & 0
\end{array}\right]\left[\begin{array}{l}
q_{i}^{k} \\
a_{i}^{k}
\end{array}\right]=T_{i} u
$$




\subsubsection{Linear Pool Model}

After the boundary addition, the pool linear model is given as,

$$
\begin{aligned}
\mathbf{X}(k+1) & =A \mathbf{X}(k)+B \mathbf{U}(k)+B_{w} \mathbf{W}(k, k-1) \\
\mathbf{Y}(k) & =C \mathbf{X}(k)+D_{v} \mathbf{V}(k)
\end{aligned}
$$

where $\mathbf{X}(k)$ is the state space vector, $\mathbf{Y}(k)$ is the output, $\mathbf{U}(k)$ is the model input, $\mathbf{W}(k)$ is the state space perturbation in discharge and $\mathbf{V}(k)$ is the output perturbation.

\subsubsection{Model Analysis}

The model tunable parameters guidelines are presented bellow:

- the number of sections considered, $N$, should be a compromise between computation effort and accuracy, and fixes the space step;

- the sample time should be tuned to maintain the Courant number $C_{r}=\alpha \frac{\Delta t}{\delta x}$ (where $\alpha$ is the downstream wave velocity, $\Delta t$ is the sample time and $\Delta x$ is the space step) close to unity. This is the same to say that the space and time resolution should be equivalent;

- for the discretization parameters, the $\phi=0.5$ is imposed to work in the centered configuration that is known to be unconditionally stable for $\theta \geq 0.5$. In particular, $\theta$ should be chosen $\theta>0.5$ to introduce numerical diffusion to eliminate the numerical oscillations introduced by the numerical method.

The model also allows for some hydraulic parameters calibration, namely the Manning hydraulic coefficient and the gates discharge coefficient.

\subsection{Hydraulic Structures}

The flow in open-channel networks is usually controlled by hydraulic structures. For the irrigation application, these structures are usually gates. These gates can be classified as overshot gates, with the flow over the gate, or undershoot gates, with the flow under the gate. Only considering free flow conditions for the first type and submerged flow conditions for the last one (the usually conditions in this type of canals) the gate equations are respectively (Laycock, 2007),

$$
\begin{aligned}
& Q_{g}=c d \cdot L_{g} \cdot \sqrt{2 g}\left(Y_{u}-Y_{g}\right)^{\frac{3}{2}} \\
& Q_{g}=c d \cdot A_{g} \cdot \sqrt{2 g} \sqrt{Y_{u}-Y_{d}}
\end{aligned}
$$

where $c d$ is the gate discharge coefficient, $A_{g}$ is the gate submerged orifice, $L_{g}$ is the gate top width and $Y_{g}$ is the gate height.

\subsection{Reservoirs}

The reservoirs are the nodes in a water conveyance network. They exhibit an integral behavior and the reservoir water depth can be modeled by the following difference equation (Moudgalya, 2007),

$$
h(k+1)=h(k)+\frac{T_{S}}{A_{S}} q_{i}(k)-\frac{T_{S}}{A_{S}} q_{o}(k)
$$

where $T_{S}$ means the sample time, $A_{S}$ the superficial area, $q_{i}(k)$ the inflow and $q_{o}(k)$ the outflow.

\section{MATLAB-SIMULINK TOOLBOX-LIBRARY}

A MatLab ${ }^{\complement}$-Simulink ${ }^{\complement}$ Toolbox-Library has been developed. It is a two stage product. In MatLab ${ }^{\complement}$ the pool models are created and in Simulink ${ }^{\complement}$ the elementary components are available as blocks for creating canal configurations. The toolbox was developed with special attention to create a flexible and modular product. The elementary blocks (pools, gates and reservoirs) are available in the library and by interconnecting them it is possible to create different canal configurations. Using different canal configurations, a water conveyance network can be created.

The Library is divided into five categories:

Pool Models: beyond the linear pool model presented also a simplified infinite dimension pool model named Integrator Delay Zero (Litrico and Fromion, 2004) (IDZ) is available;

Standard Canal Configurations: some typical canal configurations are made available: for one, two and four pools configurations;

Gates: the overshot and undershot gate equations are implemented for the sections considered above. It makes use of the geometry component for computing the gate discharge. Expansion to other geometries is made through a simple parameter change - in the cross section or top width;

Hardware: in this section some hardware static relations are available. The gate dynamics is defined through both saturation in amplitude and variation. The valves controlling the canal discharges, canal intake and offtakes, are sufficiently well approximated by a first order system with a time delay;

Geometry: computes the hydraulic cross section parameters for different geometries, to know: area, wetted perimeter, hydraulic radius, top width and hydraulic depth. 
SIMULTECH 2011 - 1st International Conference on Simulation and Modeling Methodologies, Technologies and Applications

In Figure 2 is represented the two pool canal configuration. As this is a MatLab ${ }^{\complement}-$ Simulink $^{\complement}$ ToolboxLibrary all canals composed by two pools use the same Simulink ${ }^{\complement}$ model, they only differ on the geometric characteristics in pools and gates leading to distinct dynamics calculated by the MatLab ${ }^{\circledR}$ Toolbox.

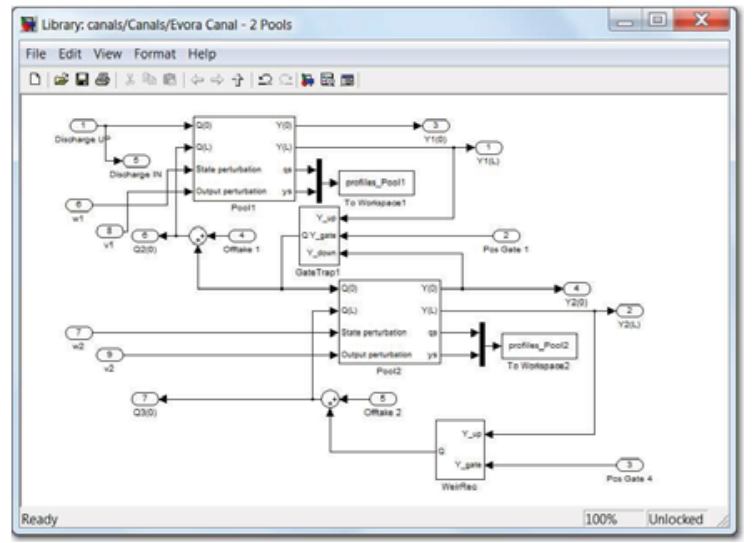

Figure 2: General view for a two pool configuration canal.

\section{EXPERIMENTAL RESULTS}

A simulator for the used experimental canal was built using the toolbox presented. For model validation, the canal was considered divided into two pools, which is equivalent to say that gates 1 and 3 are totally opened. In this configuration it is possible to proceed with water extraction along the canal pools axis which is relevant to validate the model.

The interaction with the canal is done through 7 inputs namely Figure $3, u_{1}$ the upstream inflow, $u_{2}$ gate elevation for upstream pool, $u_{3}$ gate elevation for downstream pool, $d_{1}$ offtake located upstream the gate $u_{2}, d_{2}$ offtake located upstream the gate $u_{3}, w_{1}$ outflow at upstream pool center, $w_{2}$ outflow at downstream pool center. The model outputs was chosen as the center $\left(y_{1}, y_{3}\right)$ and downstream water depth $\left(y_{2}\right.$, $\left.y_{4}\right)$ in each pool.

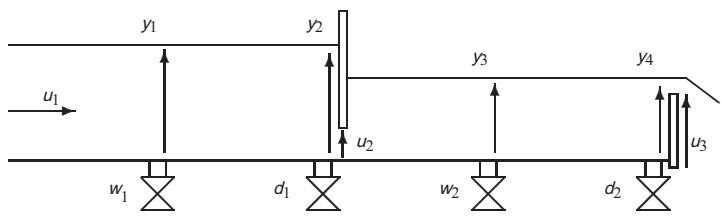

Figure 3: Schematics of the complete facility.

The linear pool model has the following numerical parameters, $N=10, \Delta x=\frac{L_{i}}{10}, \Delta t=3.3 \mathrm{~s}, \theta=0.6$ and $\phi=0.5$. Several tests were made for this canal configuration; Test $A$ : sequence with $u_{1}, u_{2}$ and $u_{4}$, about $2700 \mathrm{~s}$; Test $B$ : step sequence in $w_{1}$ and $w_{3}$, about 3240s; Test $C$ : short sequence with all inputs, about 6000 s; Test $D$ : long sequence with all inputs manipulated Figure 4, about 26000s. The model performance is quantified through the Variance Accounted For (VAF) and Root Square Error (RSE), Table $1-2$.
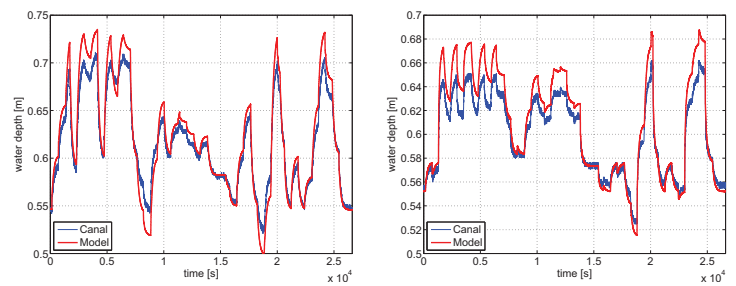

(a) Downstream water level for (b) Downstream water level for pool $1 y_{2}$.

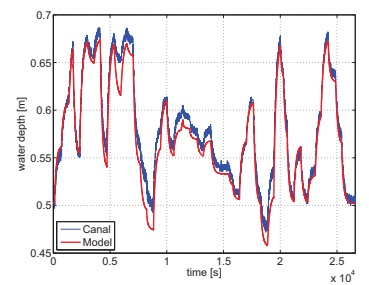
pool $2, y_{4}$.

(c) Center water depth at upstream pool $y_{1}$.

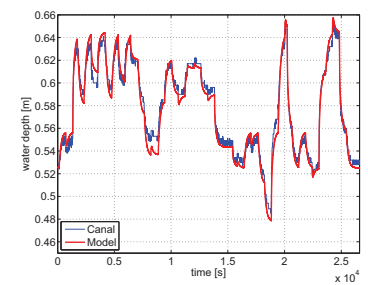

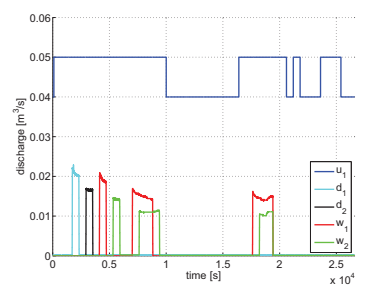

(e) Input discharges. (d) Center water depth at downstream pool $y_{3}$.

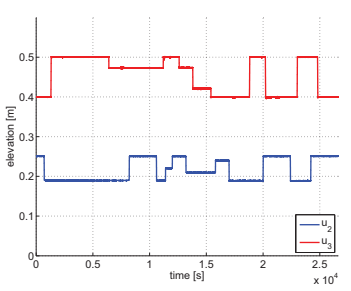

(f) Gates elevation.
Figure 4: White box model validation for Test D.

Table 1: VAF criteria for the considered water depths.

\begin{tabular}{|l|l|l|l|l|}
\hline VAF & $y_{1}$ & $y_{2}$ & $y_{3}$ & $y_{4}$ \\
\hline \hline Test A & 86.61 & 75.19 & 97.52 & 93.68 \\
Test B & 92.83 & 89.86 & 83.26 & 73.14 \\
Test C & 95.45 & 89.30 & 98.11 & 87.35 \\
Test D & 97.29 & 91.41 & 97.22 & 88.71 \\
\hline
\end{tabular}

Table 2: RSE criteria for the considered water depths.

\begin{tabular}{|l|l|l|l|l|}
\hline RSE & $y_{1}$ & $y_{2}$ & $y_{3}$ & $y_{4}$ \\
\hline \hline Test A & 0.018 & 0.017 & 0.005 & 0.008 \\
Test B & 0.009 & 0.011 & 0.005 & 0.006 \\
Test C & 0.008 & 0.013 & 0.005 & 0.012 \\
Test D & 0.013 & 0.015 & 0.007 & 0.015 \\
\hline
\end{tabular}


The best water depth VAF corresponds to center pool localizations. Upstream each gate the observed VAF decrease is explained for the gate dynamics accuracy, in particular at the downstream overshot gate. The water depth upstream the overshot gate is strongly influenced by the existing leak. Naturally, the leak intensity is a function of the water depth and this is the reason why the absolute error increases for higher water depths Figure 4(b). As the pools are in a backwater mode, the water depth error is attenuated when moving upstream as the water depth is tending to the normal depth.

Finite difference methods usually require a high space resolution to guarantee a good performance. For the model proposed this is equivalent to grow the $N+1$ number of sections considered for a pool. The important issue is to have some information about a good tradeoff between computational cost and model accuracy. To this end the simulator was tested considering different number of sections for the pool, in particular $N=\left[\begin{array}{lll}10 & 20 & 30\end{array}\right]$. The models comparison performance is done for 1800 s test duration, Table 3. As expected, the computational time increases with the space resolution but the model performance is similar. A good performance is achieved for a low space resolution which means that the pool model dimension stays tractable.

Table 3: Computational cost for a 1800s test duration.

\begin{tabular}{|l|c|c|c|}
\hline MAE & $N=10$ & $N=20$ & $N=30$ \\
\hline \hline Time [s] & 2.5 & 14 & 41 \\
$V A F_{y_{1}}$ & 94.67 & 92.62 & 91.77 \\
$V A F_{y_{2}}$ & 94.23 & 94.18 & 94.15 \\
$V A F_{y_{3}}$ & 66.29 & 62.65 & 60.75 \\
$V A F_{y_{4}}$ & 71.59 & 71.57 & 71.55 \\
\hline
\end{tabular}

\section{CONCLUSIONS}

A flexible framework toolbox for constructing water conveyance networks has been presented and validated on an experimental automatic canal. The augmented model representation for the transportation element although based on a finite difference scheme offers a good performance for low space resolution. The purposed model opens new research directions. The ability to account for water extraction along the pool can support the development of leak detection and localization algorithms. The pool monitoring capacity allows also for observer design.

\section{ACKNOWLEDGEMENTS}

This work was supported by the Portuguese Government, through Fundação para a Ciência e a Tecnologia, under the project PTDC/EEACRO/102102/2008 - AQUANET, IDMEC.

\section{REFERENCES}

Akan, A. O. (2006). Open Channel Hydraulics. Elsevier.

Duarte, J., Rato, L., Shirley, P., and Rijo, M. (2011). Multiplatform controller interface for scada application. In IFAC World Congress (Accepted in), Milan, Italy.

Laycock, A. (2007). Irrigation Systems: design, planning and construction. CAB International.

Litrico, X. and Fromion, V. (2004). Simplified modeling of irrigation canals for controller design. Journal of Irrigation and Drainage Engineering, 130:373-383.

Litrico, X. and Fromion, V. (2009). Modeling and Control of Hydrosystems. Springer-Verlag.

Moudgalya, K. (2007). Digital Control. Wiley.

Nabais, J. and Botto, M. A. (2011). Linear model for canal pools. In 8th Internation Conference on Informatics in Control, Automation and Robotics (Accepted in), Noordwijkerhout, The Netherlands.

Negenborn, R., van Overloop, P.-J., Keviczky, T., and de Schutter, B. (2009). Distributed model predictive control of irrigation canals. Networks and Heterogeneous Media, 4(2):359-380.

Szymkiewicz, R. (2010). Numerical Modeling in Open Channel. Springer-Verlag.

Weyer, E. (2001). System identification of an open water channel. Control Engineering Practice, 9:1289-1299.

Zhuan, X. and Xia, X. (2007). Models and control methodologies in open water flow dynamics: A survey. In 8th IEEE Africon Conference, pages 1-7, Windhoek, Namibia. 